\title{
Pregnancy in HIV-Positive Patients: Effects on Vaginal Flora
}

\author{
Cristina Vallone, ${ }^{1}$ Giuliano Rigon, ${ }^{1}$ Valeria Lucantoni, ${ }^{1}$ \\ Lorenza Putignani, $^{2}$ and Fabrizio Signore ${ }^{1}$ \\ ${ }^{1}$ Department of Obstetrics and Gynaecology, San Camillo-Forlanini Hospital, Piazza Carlo Forlanini 1, 00151 Rome, Italy \\ ${ }^{2}$ Parasitology Unit, Bambino Gesù Children's Hospital, IRCCS, Piazza Sant'Onofrio 4, 00165 Rome, Italy
}

Correspondence should be addressed to Fabrizio Signore, fsignore@scamilloforlanini.rm.it

Received 8 February 2012; Accepted 17 March 2012

Academic Editor: Francesco De Seta

Copyright ( $) 2012$ Cristina Vallone et al. This is an open access article distributed under the Creative Commons Attribution License, which permits unrestricted use, distribution, and reproduction in any medium, provided the original work is properly cited.

\begin{abstract}
A high proportion of HIV-infected pregnant women present pathogenic organisms in their lower genital tract. This has been associated with the development of postpartum morbility, HIV transmission to the partner and offspring, and other gynaecological conditions, such as cervical dysplasia or cancer. Vaginal flora alterations can range from $47 \%$ in Western countries to $89 \%$ in Africa in pregnant HIV-positive patients, much higher than about $20 \%$ of the general population. Pathogen organism retrieval is high. As peripartum complications due to vaginal infections seem higher in HIV-positive patients, accurate investigation and treatment of such infections are strongly mandatory.
\end{abstract}

\section{Introduction}

The classification system, drawn by the Centers for Disease Control and Prevention (CDC) of HIV infection, includes several gynaecological conditions such as persistent, frequent, or poorly responsive episodes of vaginal candidiasis, from light to severe cervical intraepithelial neoplasia (CIN), pelvic inflammatory disease (PID), chronic herpes simplex virus ulcers, and invasive cervical cancer. Three of these conditions may have a strong impact on pregnancy, namely, lower genital tract neoplasia, sexually transmitted infections and vaginitis [1].

Vaginal infections during pregnancy in HIV-positive patients have been deeply investigated in some recent studies. Preliminary data indicate an association between vaginal infections, and perinatal morbility.

\section{Materials and Methods}

Pertinent international literature was reviewed; however, due to the scarcity of studies on the matter, a systematic review resulted very difficult. We focused our attention especially on HIV-positive pregnant women. All data concerning vaginal infections during pregnancy in HIV-positive patients were thoroughly analysed.

Data concerning lower genital tract intercurrent pathogens, risks of infection, perinatal complications of vaginal infections, risks of associated conditions, effects on obstetric management, and results of prevention and therapy are discussed.

We report our preliminary findings on 54 pregnant HIV patients. Vaginal cultures were performed during the first and third trimester of pregnancy.

Cultures included tests for chlamydia, mycoplasma gram positive and negative bacteria, candida. A smear was performed for protozoa identification, notably trichomonas.

\section{Results and Discussion}

3.1. Pathogenic Lower Genital Tract Organisms in HIV Pregnancy. A study from a high-risk USA population on 854 HIV-infected women and 434 controls reports that the prevalence of bacterial vaginosis was $47 \%$ in the HIV-positive women compared with $44 \%$ in the HIV-negative women: this difference was not statistically significant $(P=0.36)$ [2]. After adjustment for other covariates, HIV-positive 
women were at higher risk of contracting bacterial vaginosis compared to HIV-seronegative women (odds ratio (OR) 1.31; $95 \%$ confidence interval (CI) $1.01-1.70$ ) by Gram's stain but not by clinical criteria (OR 1.16; CI 0.87-1.55). Among HIV-positive women, the use of antiretroviral drugs was associated with a lower prevalence of bacterial vaginosis (adjusted OR 0.54; CI 0.38-0.77). These high rates of bacterial vaginosis are similar to the ones reported in three studies carried out on women attending sexually transmitted disease centers (range 12-61\%) but are generally higher compared to healthy pregnant women (range 10-32\%).

A study conducted in Cameroon compares first trimester screening cultures from $198 \mathrm{HIV}$-positive pregnant patients to 1810 controls [1]. All lower genital tract infections, except candidiasis, were more prevalent among HIV-positive compared to HIV-negative women: vaginal candidiasis $(36.9 \%$ versus $35.4 \% ; P=0.678)$, Trichomoniasis $(21.2 \%$ versus $10.6 \% ; P<0.001)$, gonorrhoea $(10.1 \%$ versus $2.5 \% ; P<$ $0.001)$, bacterial vaginosis $(21.2 \%$ versus $15.2 \%$; $P=0.026)$, syphilis $(35.9 \%$ versus $10.6 \% ; P<0.001)$, and Chlamydia trachomatis (38.4\% versus $7.1 \%$; $P<0.001)$.

Leroy et al. reported a similar prevalence of vaginal candidiasis among HIV-infected pregnant women (22.3\%) and noninfected pregnant women (20.1\%) [3].

In South Africa a recent study on 418 HIV-infected and 383 uninfected women delivered vaginally reports that $54.8 \%$ of women had positive cultures at birth (439/801), more among HIV-infected patients compared to the uninfected ones (60\% versus $49.1 \%, P=0.002)$ [ 4 ].

According to Joao et al., the overall anogenital prevalence of GBS colonization was 49/158 (31.0\%) in a cohort of HIV-positive pregnant women during the third trimester of pregnancy [5].

Genital tract infections such as Neisseria Gonorrhoea, Chlamydia trachomatis, Candida albicans, and Trichomonas vaginalis infection have been reported to be more common in HIV-infected women (WHO/RHT/98.24 (World Health Organization), UNAIDS/98.44 (United Nations Program on HIV/AIDS)). African studies report a higher incidence of syphilis in HIV-positive women. Concurrent infection with syphilis was shown in 33\% of HIV-positive pregnant patients in South Africa, three times higher than the rate in HIVseronegative women.

3.2. Reasons for Common Vaginal Infections in HIV Patients. The prevalence of vaginal candidiasis in HIV-infected women depends on CD4 count. Burns et al. reported a 3-fold increase in vaginal candidiasis among HIV-infected women with low CD4 counts compared to HIV-infected women with normal CD4 count during pregnancy [6]. Apart from Candida spp., we reported an increase in the prevalence of bacterial vaginosis among HIV pregnant women. Bacterial vaginosis increases susceptibility to HIV infection and other genital tract pathogens, but it is not clear whether HIV increases the risk of developing bacterial vaginosis [7].

Potential biological mechanisms for this inter-relationship of sexually transmitted diseases (STDs), vaginal infection and HIV, include increased shedding of the virus in genital fluids, recruitment of HIV target cells or HIV-infected cells into the genital tract as part of the inflammatory process, stimulation of immune response to an STD causing increased viral replication, and disruption of protective epithelial barriers [8]. Direct evidence on the association of STDs with HIV was also provided by a large randomized communitybased trial in Tanzania which showed that a better targeted treatment of STDs can lower HIV incidence by about $40 \%$.

Depletion of lactobacilli may limit the production of hydrogen peroxide. It has been postulated that low vaginal $\mathrm{pH}$ inhibits CD4 lymphocyte activation and reduces HIV target cells in the vagina. Elevated vaginal $\mathrm{pH}$ may enhance HIV adherence to vaginal eukaryotic cells.

3.3. Risk of Vaginal Infection in HIV Patients. According to $\mathrm{Mbu}$ et al. [1], there is evidence that the severity of preinvasive cervical lesion is related to the degree of immunosuppression, suggesting that it contributes (at least in part) to the risk of developing preinvasive lesions $[9,10]$. Some studies suggest that the high prevalence of preinvasive cervical lesions may be related to concomitant risk factors related to the mode of HIV transmission [11]. Ahr et al. reported that HIV-positive women with low CD4 count had a higher prevalence of human papilloma virus (HPV) and that preinvasive and invasive cervical lesions of the cervix were more frequent in women with HPV [12], recognized to be a causative agent for such lesions.

3.4. Perinatal Complications of Vaginal Infections. As reported in several studies carried out in South Africa, vaginal pathogens were more common in HIV-infected women. Women with positive cultures had slightly higher rates of infectious morbidity than those negative cultures $(20.5 \%$ versus $15.2 \%, P=0.052)$. Trichomonas vaginalis and Group $B$ Streptococcus were significantly associated with sepsis $(P=$ 0.023 and $P<0.001$, resp.), whereas the presence of Candida species seemed to be protective (relative risk (RR) $0.69, P=$ $0.014)$.

Women with positive cultures had slightly higher rates of infectious morbidity than those without $(20.5 \%$ versus $15.2 \%, P=0.052)$. Trichomonas vaginalis and Group $B$ Streptococcus were significantly associated with sepsis [4].

In the past bacterial vaginosis had been considered strongly related to perinatal complications [8]. A metaanalysis reported a nonsignificant association of the treatment of bacterial vaginosis in pregnancy with the reduction in perinatal mortality [13]. In their review Kenyon et al. studied the use of antibiotics for preterm premature rupture of the membrane (pPROM) and underlined a statistically significant $43 \%$ reduction in risk of chorioamnionitis $(\mathrm{RR}=$ 0.57; 95\% CI: 0.37-0.86) [14]. However, there was no impact on perinatal mortality or fetal death before discharge $(\mathrm{RR}=0.90 ; 95 \% \mathrm{CI}: 0.74-1.10)$. Another Cochrane review by Flenady and King on antibiotics for PROM at or near term showed no impact of antibiotics on chorioamnionitis 
$(\mathrm{RR}=0.60 ; 95 \% \mathrm{CI}: 0.30-1.18)$ nor on perinatal mortality $(\mathrm{RR}=0.98 ; 95 \%$ CI: 0.14-6.89) [15].

3.5. HPV Infection in HIV-Positive Pregnant Patients. Pap smears in an African population show that HIV-positive women are more likely to have preinvasive cervical lesions: low-grade squamous intraepithelial lesion (SIL) (18.2\% versus $4.4 \% ; P<0.001$ ) and high-grade squamous intraepithelial lesion $(12.1 \%$ versus $1.5 \% ; P<0.001)[1]$. This is an 8 -fold increase in high-grade SIL among HIV-infected pregnant women compared to their noninfected counterparts. A 5-fold increase in cervical intraepithelial neoplasia among HIV-infected women was described in previous studies [16].

3.6. Maternal HIV Seroconversion and Vaginal Infection. A study by Taha on 1196 pregnant women, followed antenatally for a median of 3.4 months, reports that 27 women seroconverted by the time of delivery [17]. Postnatally, 97 seroconversions occurred among 1169 seronegative women who were followed for a median of 2.5 years. Bacterial vaginosis was significantly associated with antenatal HIV seroconversion (adjusted OR $=3.7$ ) and postnatal HIV seroconversion (adjusted OR $=2.3$ ). The approximate attributable risk of bacterial vaginosis alone was $23 \%$ for antenatal HIV seroconversions and $14 \%$ for postnatal seroconversions. The same study confirms the findings of earlier cross-sectional studies which reported associations between bacterial vaginosis and HIV among sex workers in Thailand, rural women in the Rakai district of Uganda, and urban women in Malawi. The temporal nature of such associations in this study strengthens the inference that the relationship between bacterial vaginosis and HIV acquisition could be causal. Gonorrhea, Syphilis, and Trichomoniasis were significantly associated with HIV seroconversion either in univariate or multivariate models. Gonorrhea and Syphilis showed large relative risks, although their incidence and prevalence rates were not as high as those of bacterial vaginosis or Trichomoniasis, the latter being associated with a 2-6-fold increase in risk of HIV transmission [18].

3.7. Treatment and Perinatal Benefits. The use of antiseptic or antiviral agents to cleanse the birth canal during labour and delivery has been hypothesised as a possible approach to reducing intrapartum transmission of HIV (WHO/RHT/98.24 UNAIDS/98.44). The efficacy of the use of chlorhexidine lavage to reduce the transmission of Group $B$ Streptococcus was demonstrated in Scandinavian studies. A Malawian quasirandomised study compared four-hourly aqueous chlorhexidine $0.25 \%$ solution by vaginal swabbing after vaginal examinations and a chlorhexidine wash for the baby, with a control group receiving no wash. No overall reduction was shown in the rate of HIV transmission in the above study group. There was a significant reduction in transmission in mothers who had ruptured membranes for more than four hours. Significant reductions in neonatal and puerperal sepsis were also seen following this intervention. Benzalkonium Chloride has been suggested as an alternative antiseptic agent.

\section{Conclusions}

It is not clear whether HIV infection increases the risk of acquisition of vaginal infections, but these conditions are common among sexually active women. Sexually transmitted infections (STIs) and preinvasive cervical lesions were found to be more prevalent among HIV-infected pregnant women compared to their noninfected counterparts [1]. Even comparing African and USA cohorts, HIV pregnant patients present a higher prevalence of vaginal infections compared to HIV seronegative. Women with positive vaginal cultures had slightly higher rates of infectious morbidity than those without (20.5\% versus $15.2 \%, P=0.052)$. Trichomonas vaginalis and Group B Streptococcus were significantly associated with sepsis. There is some evidence of possible benefits due to the identification and treatment of vaginal intercurrent infection in HIV-positive patients. Perinatal mortality has not been affected, but some reduction in amnionitis in case of membrane rupture has been demonstrated [13].

According to WHO guidelines, we believe that HIVpositive women should undergo a full physical examination at the first visit.

Particular attention has to be paid to any signs of vaginal thrush or lymphadenopathy. Clinical diagnosis and treatment of vaginal or cervical inflammation, abnormal discharge or STD, should be a priority. A cervical smear has to be performed if this has not been undertaken within the recent past. Colposcopy should be reserved for women who have an abnormal cervical smear result.

Prolonged rupture of membranes should be avoided, as mother-to-child transmission increases where membranes are ruptured for more than four hours [19]. Artificial rupture of membranes should not be performed if progress of labour is adequate. As a general rule, any procedure which breaks the baby's skin or increases the baby's contact with the mother's blood-such as scalp electrodes or scalp blood samplingshould be avoided unless absolutely necessary. Episiotomy should not be performed routinely, but reserved for those cases with an obstetrical indication.

Forceps may be preferable to vacuum extraction, given the risk of microlacerations of the scalp from the vacuum cup. There is an increasing evidence that elective caesarean section may help prevent HIV transmission to the newborn [20]. The operation carries risks of maternal complications and is associated with higher postoperative morbidity in HIV-positive women [21]. Prophylactic antibiotics should be administered for both elective and emergency caesarean sections.

The postpartum care of HIV-positive women should be similar to that for uninfected patients.

HIV-positive women are more prone to postpartum infectious complications, including urinary tract, chest, episiotomy, and caesarean section wound infections. All mothers should be given instructions on perineal care and the safe handling of lochia and blood-stained sanitary pads or materials.

Mothers should be counselled on the need for follow-up care, and advices on contraception systems should be given. 
On the basis of our preliminary observations, 15 out of our 54 pregnant HIV-patients showed clinical signs of vaginitis which was far in excess of the rate in our control population; a third of the HIV patients developed clinical vaginitis versus a $5 \%$ expected rate in our general population.

Common candida was by far the most represented pathogen present in almost all cases as a principal or associated agent.

As a result of our investigation, we can state that in our population an abnormal vaginal flora in a pregnant HIVpatient is a very common occurrence. Therefore, a strict screening is mandatory. Our aim is to provide full breakdown of our experience in the near future.

\section{Acknowledgment}

This paper was supported by a Grant of "Fondazione Enrico ed Enrica Sovena" to C. Vallone.

\section{References}

[1] E. R. Mbu, E. J. Kongnyuy, F. X. Mbopi-Keou, R. N. Tonye, P. N. Nana, and R. J. I. Leke, "Gynaecological morbidity among HIV positive pregnant women in Cameroon," Reproductive Health, vol. 5, no. 1, article 3, 2008.

[2] D. Warren, R. S. Klein, J. Sobel et al., "A multicenter study of bacterial vaginosis in women with or at risk for human immunodeficiency virus infection," Infectious Diseases in Obstetrics and Gynecology, vol. 9, no. 3, pp. 133-141, 2001.

[3] V. Leroy, A. de Clercq, J. Ladner, J. Bogaerts, P. van de Perre, and F. Dabis, "Should screening of genital infections be part of antenatal care in areas of high HIV prevalence? A prospective cohort study from Kigali, Rwanda, 1992-1993," Genitourinary Medicine, vol. 71, no. 4, pp. 207-211, 1995.

[4] H. M. Sebitloane, J. Moodley, and T. M. Esterhuizen, "Pathogenic lower genital tract organisms in HIV-infected and uninfected women, and their association with postpartum infectious morbidity," South African Medical Journal, vol. 101, no. 7, pp. 466-469, 2011.

[5] E. C. Joao, M. I. Gouvêa, J. A. Menezes et al., "Group B Streptococcus in a cohort of HIV-infected pregnant women: prevalence of colonization, identification and antimicrobial susceptibility profile," Scandinavian Journal of Infectious Diseases, vol. 43, no. 9, pp. 742-746, 2011.

[6] D. N. Burns, R. Tuomala, B. H. Chang et al., "Vaginal colonization or infection with Candida albicans in human immunodeficiency virus-infected women during pregnancy and during the postpartum period," Clinical Infectious Diseases, vol. 24, no. 2, pp. 201-210, 1997.

[7] E. St. John, D. Mares, and G. T. Spear, "Bacterial vaginosis and host immunity," Current HIV/AIDS Reports, vol. 4, no. 1, pp. 22-28, 2007.

[8] T. E. Taha, R. H. Gray, N. I. Kumwenda et al., "HIV infection and disturbances of vaginal flora during pregnancy," Journal of Acquired Immune Deficiency Syndromes and Human Retrovirology, vol. 20, no. 1, pp. 52-59, 1999.

[9] A. F. Nicol, G. J. Nuovo, A. Salomão-Estevez et al., "Immune factors involved in the cervical immune response in the HIV/HPV co-infection," Journal of Clinical Pathology, vol. 61, no. 1, pp. 84-88, 2008.
[10] R. Zarcone, P. Bellini, E. Carfora et al., "Incidence of CIN in HIV-positive women," Minerva Ginecologica, vol. 50, no. 5, pp. 181-183, 1998.

[11] G. P. Parham, V. V. Sahasrabuddhe, M. H. Mwanahamuntu et al., "Prevalence and predictors of squamous intraepithelial lesions of the cervix in HIV-infected women in Lusaka, Zambia," Gynecologic Oncology, vol. 103, no. 3, pp. 1017-1022, 2006.

[12] A. Ahr, A. Rody, C. Cimposiau et al., "Cervical cancer screening of HIV-positive women: is a prolongation of the screening interval meaningful?" Zentralblatt fur Gynakologie, vol. 128, no. 5, pp. 242-245, 2006.

[13] S. Ishaque, M. Y. Yakoob, A. Imdad, R. L. Goldenberg, T. P. Eisele, and Z. A. Bhutta, "Effectiveness of interventions to screen and manage infections during pregnancy on reducing stillbirths: a review," BMC Public Health, vol. 11, supplement 3, article S3, 2011.

[14] S. Kenyon, M. Boulvain, and J. P. Neilson, "Antibiotics for preterm rupture of membranes." Cochrane Database of Systematic Reviews, no. 8, Article ID CD001058, 2010.

[15] V. Flenady and J. King, "Antibiotics for prelabour rupture of membranes at or near term.," Cochrane Database of Systematic Reviews, no. 3, Article ID CD001807, 2002.

[16] A. P. Korn and D. V. Landers, "Gynecologic disease in women infected with human immunodeficiency virus type 1," Journal of Acquired Immune Deficiency Syndromes and Human Retrovirology, vol. 9, no. 4, pp. 361-370, 1995.

[17] T. E. Taha, D. R. Hoover, G. A. Dallabetta et al., "Bacterial vaginosis and disturbances of vaginal flora: association with increased acquisition of HIV," AIDS, vol. 12, no. 13, pp. 16991706, 1998.

[18] B. van der Pol, C. Kwok, B. Pierre-Louis et al., "Trichomonas vaginalis infection and human immunodeficiency virus acquisition in African women," Journal of Infectious Diseases, vol. 197, no. 4, pp. 548-554, 2008.

[19] M. L. Newell, "Caesarean section and risk of vertical transmission of HIV-1 infection," The Lancet, vol. 343, no. 8911, pp. 1464-1467, 1994.

[20] C. Peckham and M. L. Newell, "Human immunodeficiency virus infection and mode of delivery," Acta Paediatrica, vol. 86, no. 421, pp. 104-106, 1997.

[21] A. E. Semprini, C. Castagna, M. Ravizza et al., "The incidence of complications after caesarean section in $156 \mathrm{HIV}$-positive women," AIDS, vol. 9, no. 8, pp. 913-917, 1995. 


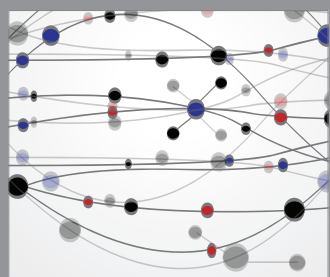

The Scientific World Journal
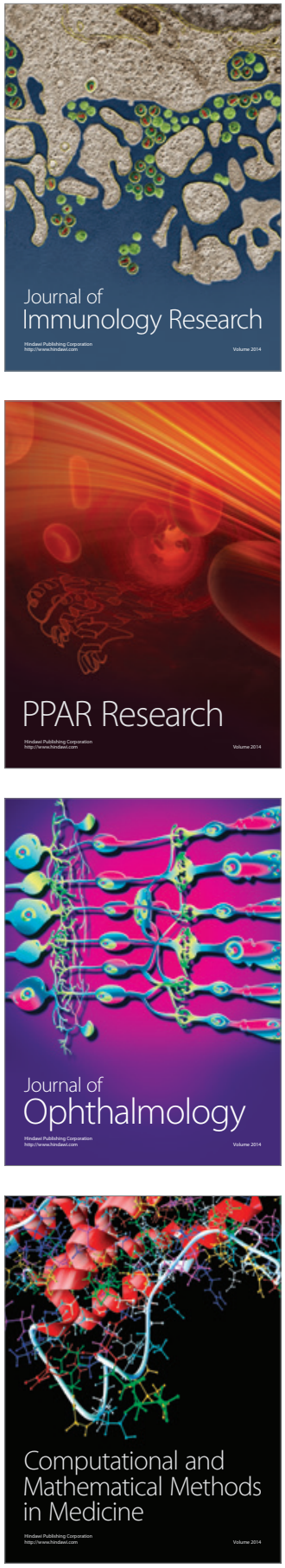

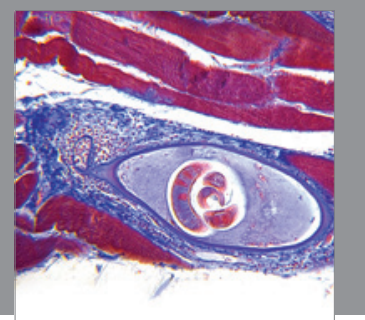

Gastroenterology

Research and Practice
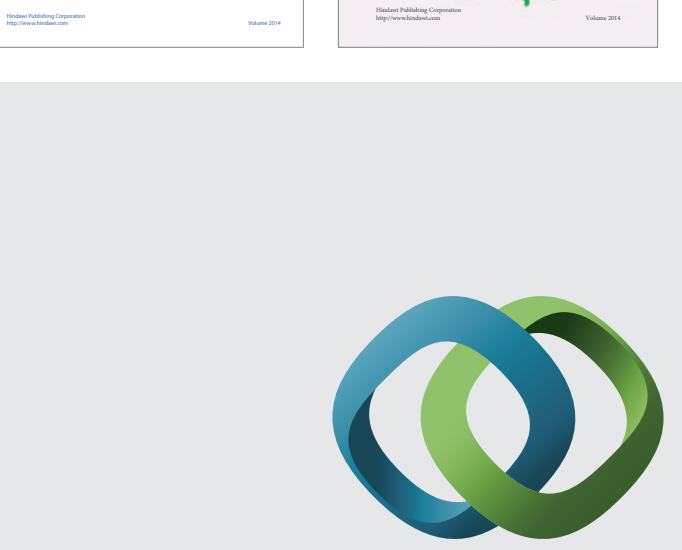

\section{Hindawi}

Submit your manuscripts at

http://www.hindawi.com
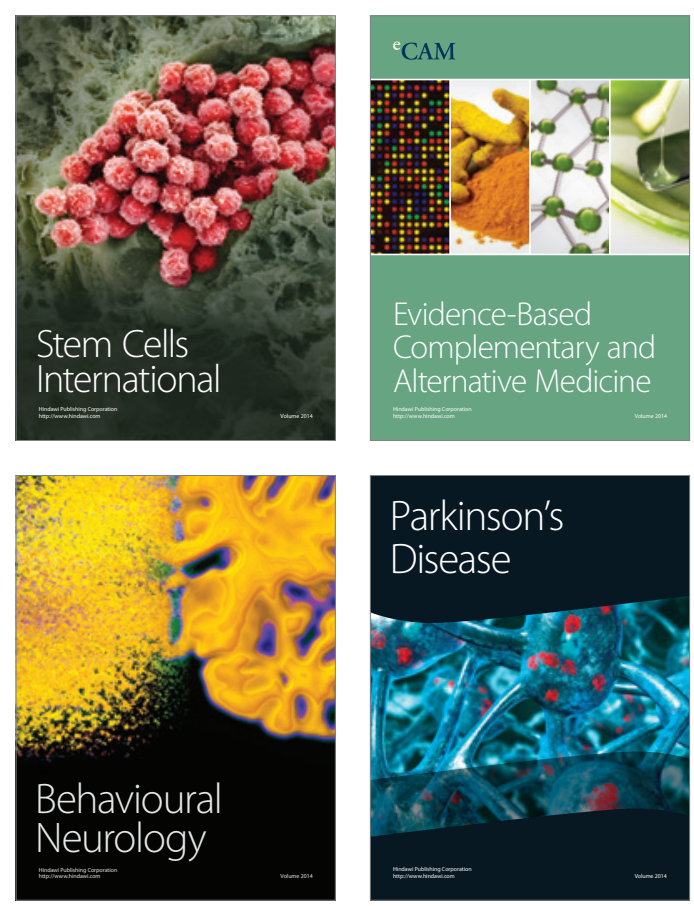

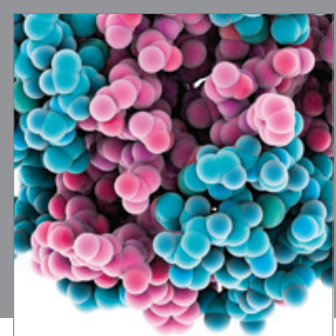

Journal of
Diabetes Research

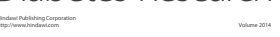

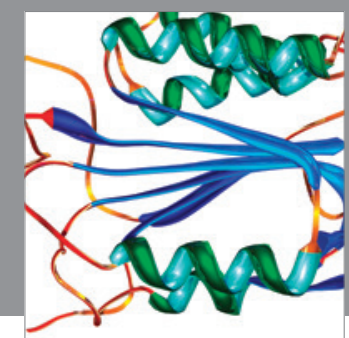

Disease Markers
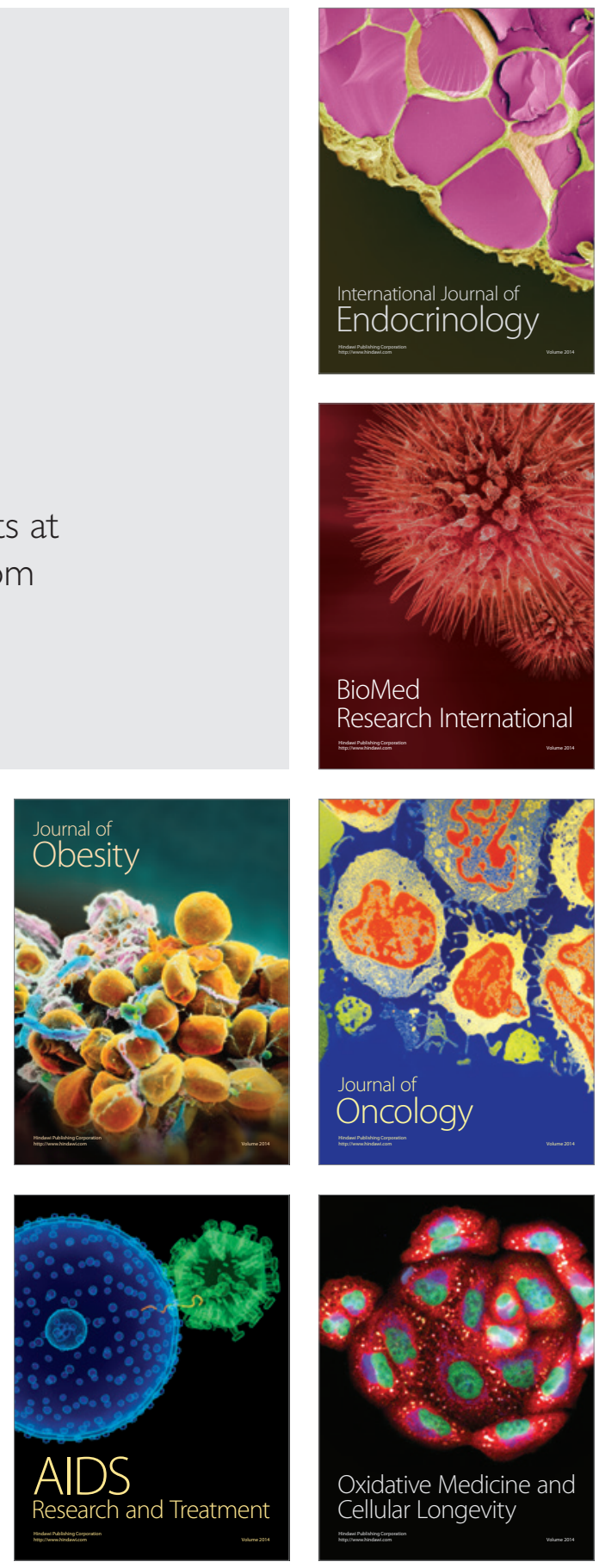